\title{
MILLIMETRE OBSERVATIONS OF GAMMA-RAY BURSTS AT IRAM
}

\author{
A.J. Castro-Tirado ${ }^{1}$, M. Bremer ${ }^{2}$, J.M. Winters ${ }^{2}$, J.C. Tello ${ }^{1}$,
} S.B. Pandey ${ }^{3}$, A. de Ugarte Postigo ${ }^{1,4}$, J. Gorosabel ${ }^{1,5,6}$, S. Guziy $^{7}$, M. Jelinek ${ }^{1}$, R. Sánchez-Ramírez ${ }^{1}$, D. Pérez-Ramírez ${ }^{8}$ and J.M. Castro Cerón ${ }^{9}$

\begin{abstract}
Since 1997, and following our detection of the first mm afterglow, we have followed-up 70 GRBs, mainly with the IRAM's Plateau de Bure Interferometer, what can be considered as the IRAM Legacy GRB Sample. 66 events were observed at $3 \mathrm{~mm}$, with 19 of them being detected (with another 3 having marginal detections). 32 GRBs were followed up at $1 \mathrm{~mm}$, with 6 of them being detected. Redshifts for the GRB afterglows lie in the range $\mathrm{z}=0.03-8.3$, with measured flux densities (at $3 \mathrm{~mm}$ ) varying between 0.25 and $60 \mathrm{mJy}$ (but usually $<1.5 \mathrm{mJy}$ ) with first observations taking place around 1-2 days after the GRB. Forward shock emission expleains the observations with the exception of one particular case (GRB 090423 at $\mathrm{z}=8.2$ ) for which reverse shock emission is required.
\end{abstract}

\section{Introduction}

Since 1997, following our detection of the first millimeter (mm) afterglow (GRB 970508. Bremer et al. 1998) we are conducting pioneering mm observations

\footnotetext{
${ }^{1}$ Instituto de Astrofísica de Andalucía (IAA-CSIC), Glorieta de la Astronomía s/n, 18008 Granada, Spain

${ }^{2}$ Institute de Radioastronomie Milimetrique (IRAM), Saint Martin d’Hères, France

${ }^{3}$ Aryabhatta Research Institute of Observational Sciences (ARIES), Nainital, India

${ }^{4}$ Dark Cosmology Centre, Niels Bohr Institute, University of Copenhagen, 2100 Copenhagen, Denmark

${ }^{5}$ Unidad Asociada CSIC-UPV/EHU, Departamento de Física Aplicada I, Escuela T. Superior de Ingeniería, Bilbao, Spain

${ }^{6}$ Ikerbasque, Basque Foundation for Science, Alameda de Urquijo 36-5, 48008 Bilbao, Spain

7 Nikolaev Astronomical Observatory, University of Nikolaev, Ukraine

8 Universidad de Jaén, Spain

${ }^{9}$ ESAC, Robledo de Chavela, Madrid, Spain
} 
of GRBs with the IRAM's Plateau de Bure Interferometer (PdBI) in the French Alps.

Observations at these wavelengths are most essential due to the following reasons: i) Negligible absorption effects (like gamma-rays), i.e. dark or ultra- high- $z$ bursts can be detected; ii) The peak of the GRB synchrotron spectrum peaks in the $\mathrm{mm}$ range; iii) No self-absorption effects (as seen at lower-frequencies); iv) Negligible interstellar scintillation effects (as seen at lower-frequencies); v) The peak of the GRB afterglow emission takes hours-days to cross the $\mathrm{mm}$ band; vi) PdBI is the only Northern Hemisphere observatory nowadays that has the sensitivity high enough to detect the mm afterglow emission for a considerable number of events (and perhaps even for detecting the forward emission too in some cases); vii) A logistics advantage: the flexibility of $\mathrm{PdBI}$ due to the fact that is a service observatory (with no observers present at the time of executing their programs) makes it easy to reschedule a target of opportunity ( $\mathrm{ToO}$ ) program.

This work enlarges the IRAM sample previously discussed (de Ugarte Postigo et al. 2012) as it does include additional datasets and the details can be seen in Castro-Tirado et al. (2013).

\section{Observations and results}

We have mainly used the PdBI (Guilloteau et al. 1992), located at $2550 \mathrm{~m}$ a.s.l. in the Haute Alpes (France). It is the most sensitive observatory in the Northern Hemisphere operating at millimetre wavelengths $(0.8,1.2,2$ and $3 \mathrm{~mm})$. In 1996 is started observations with 5 antenae, increasing to 6 as of 2002. New generation receivers were installed in 2007. In fact, the wideband correlator WIDEX, installed in 2010, has significantly increased the number of detections due to its superb sensitivity. Additional observations were carried out on a very few cases with the $30 \mathrm{~m}$ IRAM antenna at Pico Veleta (2920 m a.s.l.) in Sierra Nevada (Spain).

Following our 16 yr study of GRB afterglows, the main results are the following ones:

1. The number of follow-ups amounted to 70, with 19 detections of mm afterglows achieved at $3 \mathrm{~mm}, 6$ detections at $1 \mathrm{~mm}$, with 4 of the events being simultanously detected at 1 and $3 \mathrm{~mm}$.

2. Redshifts for the GRB afterglows lie in the range $z=0.03-8.3$, with measured flux densities (at $3 \mathrm{~mm}$ ) varying between 0.25 and $60 \mathrm{mJy}$ (but usually $<1.5 \mathrm{mJy}$ ) with first observations taking place around $1-2$ days after the GRB. We have detected 2 (out of 3) GRBs at $\mathrm{z}>6$ : GRB $050904(\mathrm{z}=6.3$ ) and $090423(\mathrm{z}=8.3)$. On the lower redshift end, we have detected the low- $\mathrm{z}$, faint GRB $090108 /$ SN 2008D $(z=0.03)$.

3. Amongst the long-duration class, we have detected four dark GRBs: GRB 051022 (associated with a $\mathrm{z}=0.809$ galaxy), GRB 090404 (whose galaxy is not properly identified), GRB 111215A (associated with a galaxy in the range $1.8<\mathrm{z}<2.5)$ and GRB 120624B. 


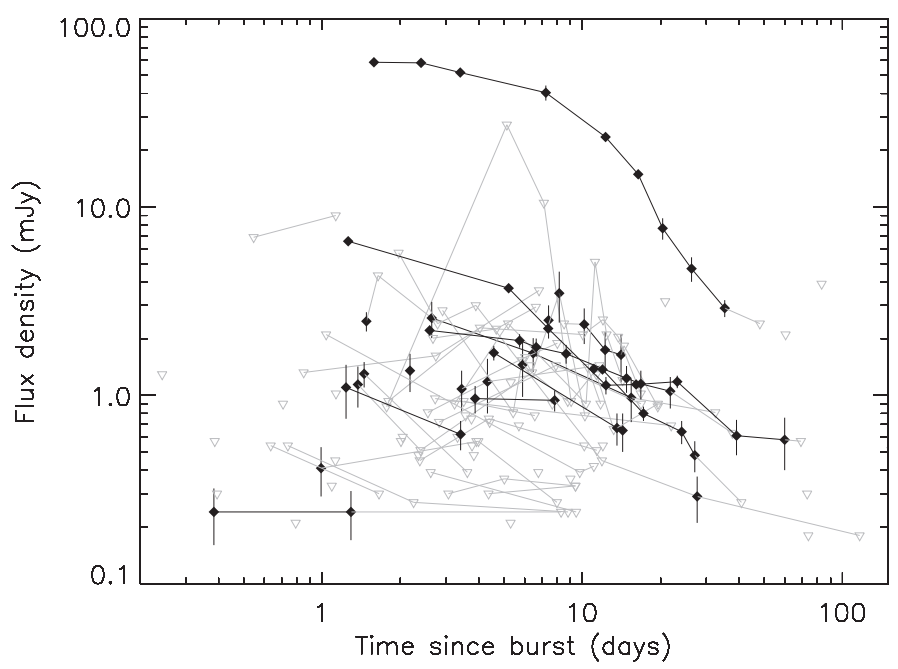

Fig. 1. The observed light curves and upper limits for 64 events observed at $3 \mathrm{~mm}$ as described in this work, with 18 of detected (black circles, filled) and 45 undetected (grey upside down triangles, empty). GRB 070610 and GRB 110328A are excluded as they have a different physical origin.

\section{Conclusions}

Millimetre observations are a powerful tool for gaining a better understanding of the GRB physics, especially the highly extinguished events and very high redshift bursts. The 23 GRBs detected imply a $32 \%$ success rate (for this non-blind sample). Millimetre observations are not affected by high-z or extinction and usually can lead to sample the synchrotron peak in the spectrum as well as detecting the tail of the prompt emission and the forward shock in one case (GRB 090423 at $\mathrm{z}=8.3)$.

We acknowledge the support of the Spanish programs AYA2009-14000-C03-01 (MICINN) and AYA2012-39727-C03-01 (MINECO). Based on observations carried out with the IRAM Plateau de Bure Interferometer and with the $30 \mathrm{~m}$ Pico de Veleta antenna. IRAM is supported by INSU/CNRS (France), MPG (Germany) and IGN (Spain).

\section{References}

Bremer, M., Krichbaum, T.P., Galama, T.J., et al., 1998, A\&A 332, L13

Castro-Tirado, A.J., Bremer, M., Winters, J.-M., et al., 2013, in press

Guilloteau, S., Delannoy, J., Downes, D., et al., 1992, A\&A 262, 624

de Ugarte Postigo, A., Lundgren, A., Martín, S., et al., 2012, A\&A, 538, A44 
